\title{
Energy transformation and energy storage in the Midwest and beyond - ADDENDUM
}

doi: https://doi.org/10.1557/mre.2019.6

The following footnote should be included in this article [1]:

This paper was commissioned and accepted for publication by Elizabeth Kocs, who served as Editor-in-Chief of this journal from 2015-2018.
Ellen Anderson

REFERENCE:

1. Anderson E. (2019). Energy transformation and energy storage in the Midwest and beyond. MRS Energy \& Sustainability, 6, E6. Cambridge University Press. 